\title{
Response of Bone Mineral Density to Physical Exercises after Thyroidectomy
}

\author{
Heba M. Mohamady ${ }^{1}$, Manar H. Abdel Sattar ${ }^{2}$, Ashraf H. Mohammed ${ }^{1}$, Ahmed M. Abdellatif ${ }^{3}$ \\ ${ }^{1}$ Department for Surgery, Faculty of Physical Therapy, Cairo , Cairo University, Egypt \\ ${ }^{2}$ Department of Radiology, Faculty of Medicine, Cairo, Cairo University, Egypt \\ ${ }^{3}$ Sheikh Zayed Central Hospital, Giza, Egypt \\ Email address: \\ heba.mohamady@yahoo.com (H. M. Mohamady)
}

\section{To cite this article:}

Heba.M.Mohamady, Manar H. AbdelSattar, Ashraf H. Mohammed, Ahmed M. Abdellatif. Response of Bone Mineral Density to Physical Exercises after Thyroidectomy. Journal of Surgery. Special Issue: Postoperative Pain Syndrome.Vol. 3, No. 2-1, 2015 , pp. $23-26$.

doi: $10.11648 /$ j.js.s.2015030201.15

\begin{abstract}
Introduction: Thyroidectomy could be associated with bone loss because of an endogenous excess of thyroxin, over enthusiastic thyroid replacement therapy following surgery, deregulation of bone resorption as consequence of calcitonin deficiency or some combination of these factors. The aim was to investigate the effect of weight bearing exercises on Improving bone mineral density in post thyroidectomy patients suffering from osteoporosis. Subjects and methods: Thirty osteoporotic patients were assigned randomly into two equal groups; their ages ranged from 20-45 years. The study group received weight bearing exercises program in addition to routine medical treatment. Control group received medical treatment only. Study group received two sessions per week for two successful months. The data were collected before and after the same period of treatment for both groups. Evaluation procedures were carried out to evaluate the bone mineral density using Dual Energy X-ray Absorptiometry. Results: Post treatment both groups showed a variant improvement in bone mineral density, but a significant improvement difference was recorded between the two groups in favor of the study group. Percentage of improvement of bone mineral density in the study group was $10.69 \%$, while it was $2.8 \%$ in the control group. Conclusion: Weight bearing exercises were considered to be an effective modality for improving bone mineral density in subjects suffering from osteoporosis following thyroidectomy.
\end{abstract}

Keywords: Thyroidectomy, Osteoporosis, Weight Bearing Exercises, Dual Energy X-Ray Absorptiometry

\section{Introduction}

Thyroidectomy could be associated with bone loss because of an endogenous excess of thyroxin, over enthusiastic thyroid replacement therapy following surgery, deregulation of bone resorption as consequence of calcitonin deficiency or some combination of these factors ${ }^{(1,2)}$. The secretion of calcitonin is dramatically reduced by total or subtotal thyroidectomy and/ or radioiodine therapy and calcitonin deficiency has also been postulated to cause osteopenia ${ }^{(3)}$.

Histomorphometric studies demonstrate that thyroid hormones increase the activation of new remodeling cycles and stimulate osteoclastic and osteoplastic activity in trabecular and cortical bone ${ }^{(4)}$.

Bone remodeling is a dynamic process and characterized by coupling between resorption and formation. It is initiated by activation of osteoclast precursors that become mature osteoclasts by proliferation and differentiation. The osteoclasts cause bone resorption until they have reached a final resorption depth. The osteoblasts then invade the area and begin bone formation ${ }^{(5,6)}$. The end product of the remodeling sequence is the bone structural unit which is characterized by a certain structural thickness ${ }^{(7)}$.

Evidences supporting the role of weight bearing exercise in bone health has accumulated from cross sectional, retrospective, prospective and intervention studies ${ }^{(8,9)}$.Bone remodeling occurs in response to physical stress, so physical inactivity can lead to significant bone loss. Weight bearing exercise can increase peak bone mass achieved in adolescence, and a highly significant correlation between bone strength and muscle strength has been determined ${ }^{(10,11)}$.

Weight bearing exercise is the exercise that makes body works against gravity. Regular weight bearing exercises in children and teenager help produce strong bone and are good 
options for promoting healthy bones ${ }^{(12)}$.Exercise may contribute to the prevention of osteoporosis and fractures by increasing the amount of bone accretion during growth, by reducing menopause related and age related bone loss, or by restoring bone already lost in the elderly ${ }^{(13,14)}$. Physical activities involving high impact or weight bearing movements provides an osteogenic stimulus that may enhance bone mass at any age. The practice of high impact or weight bearing activities in early childhood may lead to substantial gains of bone mass ${ }^{(15)}$.

\section{Subjects,MaterialsandMethods}

This study was carried out on 30 adult patients of both sexes suffering from osteoporosis after thyroidectomy, their ages ranged from 20 to 45 years and selected from endocrinal surgery and orthopedic departments at Kasr Al Ainy Teaching Hospital, Cairo University, Egypt during the period of March 2013 to November 2013.

Patients were randomly assigned into two equal groups; Group A (exercises group) and Group B (Control group). Group A received physical exercises program (weight bearing exercises in the form of weight bearing exercises, walking on treadmill, stair climbing and strengthening exercises) ${ }^{(16)}$ in addition to routine medical intervention (Pisphosphonates, calcium and vitamin D) and group B received routine medical intervention (Pisphosphonates, calcium and vitamin D) only.

Exclusion criteria: patients with the following conditions were excluded from the study; early menopause, andropause, patients had drugs that affect bone metabolism (glucocorticoids, heparin, warfarin and cyclosporine), heavy smokers, alcohol drinking, sedentary patients, and patients had any gonadal disease that affects hormonal balance.

The work has been carried out in accordance with the code of Ethics of the World Medical Association (Declaration of Helsinki) for experiments involving humans, and parents filled approval consent to share in the study.

Regarding the osteoporosis, the assessment approach was: Dual-energy x-ray absorptiometry.By measuring specific bones, usually the spine, hip, and wrist. The density of these bones was then compared with an average index based on age, sex, and size. The resulting comparison is used to determine risk for fractures and the stage of osteoporosis in an individual.

Average bone mineral density $=\mathrm{BMC} / \mathrm{W}\left[\mathrm{g} / \mathrm{cm}^{2}\right]$

- $\mathrm{BMC}=$ bone mineral content $=\mathrm{g} / \mathrm{cm}$

- $\mathrm{W}=$ width at the scanned line ${ }^{(17)}$.

- Data was graded according to Z-score.

Z-score:

The Z-score is the comparison to the age matched normal subject. This is the number of standard deviations a patient's bone mineral density (BMD) differs from the average BMD of their age, sex, and ethnicity. This value is used in premenopausal women, men under the age of 50, and in children. It is most useful when the score is less than 2 standard deviations below this normal. In this setting, it is helpful to scrutinize for coexisting illnesses that may contribute to osteoporosis such as glucocorticoid therapy, hyperparathyroidism, or alcoholism. Z-score minus 1.5 and lower is considered below expected range for age, while Zscore above minus 1.5 is considered within expected range for age ${ }^{(18)}$.

\subsection{Statistical Analysis}

Data was entered and analyzed using the student t-test and p-value set at $<0.05$ was considered significant.

\section{Results}

The statistical analysis for patient's socio-demographic data (age, sex and body mass index) revealed a non significant difference between both groups $[\mathrm{P} \geq 0.05]$.

There was a significant improvement in bone mineral density of the group A as well as for group B after 2 months of treatment $(p<0.05)$. Group A pretreatment mean value of bone mineral density was $-2.15 \pm 0.17 \mathrm{~g} / \mathrm{cm}^{2}$ according to Zscore, while it was $-1.92 \pm 0.2 \mathrm{~g} / \mathrm{cm}^{2}$ post treatment $\{P<0.05\}$. However, the mean value of bone mineral density before treatment (pretreatment)of group B was $2.14 \pm 0.19 \mathrm{~g} / \mathrm{cm}^{2}$, while it was $2.08 \pm 0.19 \mathrm{~g} / \mathrm{cm}^{2}$ post treatment $\{P<$ $0.05\}$.Results revealed that; there was no significant difference in the mean values of bone mineral density pretreatment between both groups. While the post- treatment results showed a significant difference $\{P<0.05\}$.[Table 1] and figure [1]

Table 1. Pre and post treatment results of bone mineral density for both groups.

\begin{tabular}{lllll}
\hline & \multicolumn{2}{l}{ Pretreatment } & \multicolumn{2}{l}{ Posttreatment } \\
\cline { 2 - 5 } & GroupA & GroupB & GroupA & GroupB \\
\hline$\overline{\mathrm{X}}$ & -2.15 & -2.14 & -1.92 & -2.08 \\
$\mathrm{SD} \pm$ & \pm 0.17 & \pm 0.19 & 0.2 & 0.19 \\
$\mathrm{~T}-$ value & 0.09 & & 2.2 & \\
P-value & 0.92 & 0.03 & \\
Significance & Nonsignificant & Significant \\
\hline
\end{tabular}

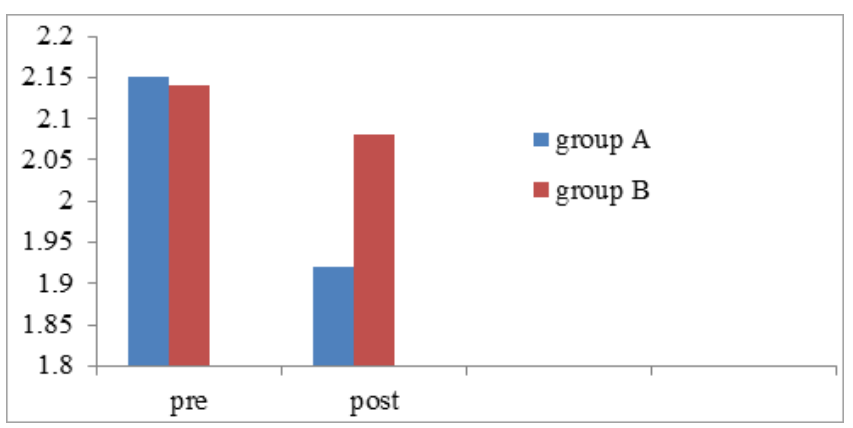

Figure 1. Mean values of pre and post treatment results of bone mineral density for both groups.

Statistical analysis of results showed a significant improvement in bone mineral density for group A as well as for group B after 2 months of treatment as revealed in table 2 and figure $2(\mathrm{p}<0.05)$. 
Table 2. Pre and post treatment results of bone mineral density for each group.

\begin{tabular}{lllll}
\hline & GroupA & GroupB \\
\cline { 2 - 5 } & Pre & Post & Pre & Post \\
\hline$\overline{\mathrm{X}}$ & -2.15 & -1.92 & -2.14 & -2.08 \\
$\mathrm{SD} \pm$ & 0.17 & 0.2 & \pm 0.19 & \pm 0.19 \\
$\mathrm{~T}-$ value & 14.14 & & 14.04 & \\
P-value & 0.0001 & & 0.0001 & \\
Significance & Significant & & Significant & \\
\hline
\end{tabular}

$\begin{array}{r}2.2 \\ 2.15 \\ 2.1 \\ 2.05 \\ 2 \\ 1.95 \\ 1.9 \\ 1.85 \\ 1.8 \\ \quad \text { group A } \quad \text { group B } \\ \hline\end{array}$

Figure 2. Mean values of pre and post treatmentresults of bone mineral density for each group.

\section{Discussion}

Transient increase in Thyroid stimulating hormone (TSH), with peak levels 3 to 6 months after the operation, has been detected after thyroidectomy ${ }^{(19)}$. In some patients early transient hypothyroidism is developed and subsides after some months. It occurs mostly in the first 3 months after the operation and subsides after 6 months ${ }^{(20)}$.

The findings of this study indicated that patients suffering from osteoporosis following thyroidectomy had a significant improvement in BMD after application of weight bearing exercises program. The statistical analysis revealed a significant improvement of osteoporosis in the exercises group than that of the control group; the percentage of improvement was $10.69 \%$ and $2.8 \%$ for the two groups respectively.

The results of this study are online with those in another work which approved that regular physical activity is cheap and safe way of both improving bone strength and reducing the risk of fall ${ }^{(21,22)}$. It is also supported by other studies which mentioned that; bone tissue responds to repeated mechanical deformation and muscular contractions, which lead to an increase in electric pulsed currents in bone via piezoelectric effects. When mechanical forces through mechanical loads during exercise are converted into electrical stimuli, specific bone tissue cells can begin the osteogenesis process resulting in an increase in $\mathrm{BMD}^{(23,24)}$.

From the gained results it could be concluded that; weight bearing exercises had significant improvements of bone mineral density in cases of osteoporosis following thyroidectomy.

\section{References}

[1] Cummings S.R, Nevitt M.C, Browner W.S, Stone K.m, Fox K.M, Ensrud K.E, Cauley J, Black D, Vogt T.M. Risk factors for hip fracture in white women: The study of osteoprotic fractures research group. N Engl J MED, 1995, 322: 267-773.

[2] Lawrence G, RaisZ.Pathogenesis of osteoporosis: Concepts, Conflicts and Prospects. J Clin Invest, 2005,115 (12): 33183325 .

[3] Mc Dermott M.T, Kidd C.T.S, Blue P, G Head V, Hofeldt F.D. Reduced bone mineral content in totally thyroidectomized patients : possible effect of calcitonin deficiency. J Clin. Endocrinal Metab, 1983, 56: 936-939.

[4] Dhanwal DK. Thyroid disorders and bone mineral metabolism. Indian J EndocrinolMetab. 2011; 15(Supp12): S107-S112.

[5] Welten D.C., Kemper H.C. and Post G.B. Assessment of fracture risk and its application to screening for postmenopausal osteoporosis. Report of a WHO Study Group. WHO technical Report Series, 1994, 843: 120-129.

[6] Valimaki M.J, Tiihonen M, Laitinen K. Bone mineral density measured by dual-energy x-ray absorptiometry and novel markers of bone formation and resorption in patients on antiepileptic drugs. J Bone Miner Res, 1994, 9: 631-637.

[7] Eriksen E.F. Normal and pathological remodeling of human trabecular bone. Three-dimensional reconstruction of the remodeling sequence in normal and in metabolic bone disease."Endocr Rev, 1986,7: 379-381.

[8] Andredi A, Monteleone M, Van Loan M, Proncenzio L, Tarantino U, De Lorenzo A. Effects of different sports on bone density and muscle mass in highly trained athletes. Med. Sci. sports Exer, 2001: 33(4): 507-511.

[9] Hara S, Yanagi H, Amagai H. Effect of physical activity during teenage years, based on type of sport and duration of exercise, on bone mineral density of young, premenopausal Japanese women. Calcif Tissue Int, 2001, 68: 23-30.

[10] Pfeifer M, Minne H.W. Vitamin D and hip fracture." Trends in Endocrinology and Metabolism, 1999, 10: 417-420.

[11] 11.Schoenau E, Werhahn E, Schiedermaier U, Mokow E, Schiessl H, Scheidhauer K, Michalk D. Influence of muscle strength on bone strength during childhood and adolescence. Hormone Research, 1996,45 (1): 63-66.

[12] Seigrist M. Role of physical activity in the prevention of osteoporosis. Mineral Res, 2008,10: 1303-1311.

[13] Beck B.R, Snow C.M.Bone health across the lifespan exercising our options. Exerc Sport Sci Rev, 2003, 31: 117122.

[14] Nikander R. Femoral neck structure in adult female athletes subjected to different loading modalities. J. Bone Min. Res, 2005,20: 520-528.

[15] Bass S.L. The prepubertal years, a uniquely opportune stage of growth. When the skeleton is most responsive to exercise?.Sports Med,2000, 30(2): 73-80.

[16] Hakan L, Anna S, Ulrika P, Gustaf B. (2005): "Wight bearing exercise and osteoporosis." Osteo Int,2005, 16: 1117-1123. 
[17] Mishra L, Mahavir B, Shanu N, Ranu S. Dental care in the patients with bisphosphonates therapy.International Journal of Dental Clinics,2011, 3 (1): 60-64.

[18] Blake G.M, Glen M, Fogelman, Ignac .Effect of bone strontium on BMD measurements. Journal of Clinical Densitometry,2007, 10 (1): 34-38.

[19] Kulaçoglu H, Dener C, Ziraman I, Kama N.A. Thyroxine prophylaxis after bilateral subtotal thyroidectomy for multinodular goiter. Endocr. J,2000, Jun.; 47 (3): 349-352.

[20] Wilmar M, Wiersinga .Hypothyroidism and myxedema coma. In: Degroot L.S. Endocrinology. $4^{\text {th }}$ ed. Saunders,2001, 30: 1494.
[21] Bischoff H.A, Dawson H.B, Willett W.C. Effect of vitamin D on falls: a meta-analysis. JAMA, 2004, 291(16): 1999-2006.

[22] De Laet C, Kanis J, Oden A, Johanson H, Johnell O, Delmas P. Body mass index as a predictor of fracture risk: a metaanalysis. Osteoporos Int,2005, 16: 1330-1338.

[23] Kogianni G, Noble B.S. The biology of osteocytes. Curr Osteoporos Rep,2007, 5(2): 81-86.

[24] Meka N, Katragadda S, Cherian B. Endurance exercise and resistance traning in cardiovascular disease. Cardiovas Disorders, 2008, 7: 21-26. 